Ann. Biol. anim. Bioch. Biophys., I975, 15 (3), 6I I-6r4.

NOTE

\title{
UTILISATION DIGESTIVE D'UNE HUILE DE HARENG PARTIELLEMENT HYDROGÉNÉE GHEZ LE RAT
}

\author{
P. O. ASTORG et G. ROCQUELIN \\ Station de Recherches sur la Qualité des Aliments de l'Homme, \\ Centre de Recherches de Dijon, I. N. R. A., \\ 7, rue Sully, \\ 21034 Dijon Cedex
}

\section{RÉSUMÉ}

L'utilisation digestive apparente d'une huile de hareng partiellement hydrogénée (contenant I3 p. roo de $\mathrm{C}_{22}: 1$ ) est inférieure à celle de l'huile d'arachide; elle est améliorée par une addition d'huile de maïs.

Les huiles de poissons partiellement hydrogénées, utilisées en margarinerie, sont riches en acides gras monoinsaturés à longue chaîne $\left(\mathrm{C}_{20}:{ }_{1}\right.$ et $\left.\mathrm{C}_{22}:{ }_{1}\right)$, isomères des acides éicosénoïque (cis- $\Delta_{11} C_{20: 1}$ ) et érucique (cis- $\Delta_{13} C_{22}: 1$ ) de l'huile de colza. Celle-ci, outre ses effets particuliers sur le myocarde, est connue pour son utilisation digestive médiocre (RocQuelin et Leclerc, I969), due principalement à l'absorption incomplète de l'acide érucique. Il nous a donc paru intéressant de comparer, du point de vue de l'utilisation digestive, une huile de hareng partiellement hydrogénée et l'huile de colza riche en acide érucique.

Vingt rats Wistar mâles provenant de l'élevage de la Station, pris au sevrage, sont placés dans des cages individuelles permettant la collecte séparée des urines et des fèces. Ils sont répartis en 4 lots de 5 et reçoivent, ad libitum, des régimes semi-synthétiques équilibrés contenant 5 p. Ioo de lipides en poids : huile d'arachide (lot témoin), huile de colza riche en acide érucique, huile de hareng partiellement hydrogénée (d'origine norvégienne), mélange d'huile de hareng partiellement hydrogénée et d'huile de maïs $\mathrm{I}_{3} / 2$ en poids. Ce quatrième lot est constitué en raison de la pauvreté de l'huile de hareng partiellement hydrogénée en acide linoléique (moins de 2 p. roo). Les teneurs des huiles en leurs principaux acides gras sont indiquées dans le tableau $\mathbf{I}$. 


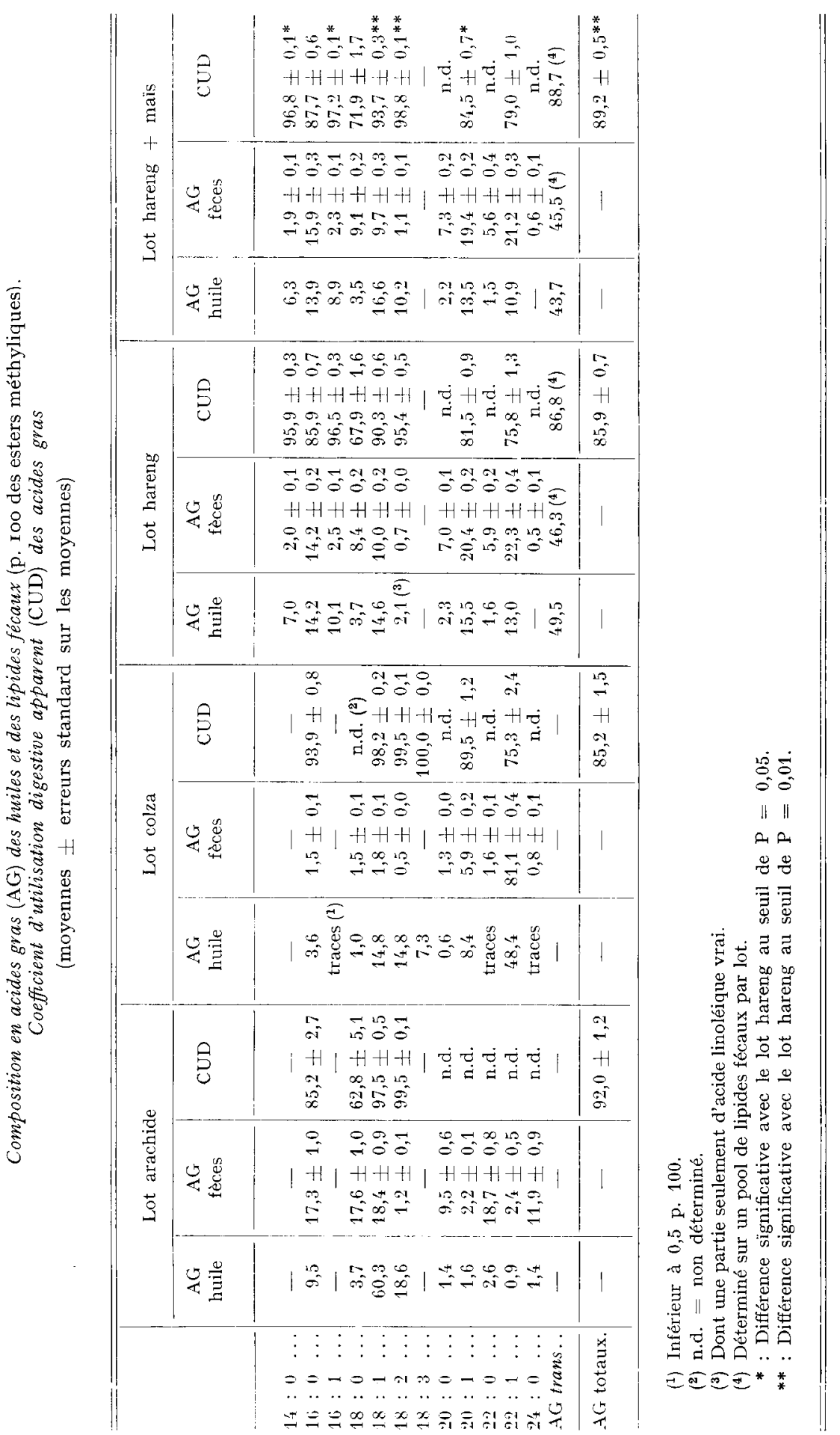


Les consommations de nourriture sont déterminées individuellement et quotidiennement par pesée. Après 24 heures d'adaptation au régime, les fèces sont collectées chaque jour, rat par rat, pendant une période de 8 jours. Les lipides fécaux sont extraits par la méthode de SAvary et Constantin (1966) et pesés; après saponification et extraction de l'insaponifiable, les acides gras des lipides fécaux sont dosés par pesée. La composition en acides gras de ces lipides est déterminée par chromatographie gaz-liquide des esters méthyliques correspondants sur phases stationnaires polaires (butanediol succinate et diéthylène glycol succinate). Le dosage des acides gras trans totaux dans les huiles et dans les lipides fécaux est réalisé par absorptiométrie dans l'infra-rouge (A. O.C. S. Tentative Method, 1973).

Les résultats confirment que l'huile de colza est absorbée moins complètement que l'huile d'arachide (tabl. 2). Il en est de même de l'huile de hareng partiellement hydrogénée. L'addition d'huile de maìs à cette dernière huile améliore son utilisation digestive. En effet, en admettant que le coefficient d'utilisation digestive apparent (CUD) ( $\left.{ }^{1}\right)$ des acides gras de l'huile de maïs est de 95 à 98 p. Ioo, celui des acides gras du mélange huile de hareng - huile de maïs $13 / 2$ atteindrait seulement $87, \mathrm{I}$ à 87,5 p. Ioo (+ I,2 à $\mathrm{I}, 6$ points par rapport à l'huile de hareng); or il est nettement plus élevé $(+3,3$ points).

\section{TABLEAU 2}

Quantités ingérées et excrétées ( $\mathrm{g} / \mathrm{rat} / 8$ jours) de matière sèche (MS), de lipides totaux (LT) et d'acides gras (AG) du régime. Coefficients d'utilisation digestive apparents (CUD)

(moyennes \pm erreurs standard sur les moyennes)

\begin{tabular}{|c|c|c|c|c|}
\hline & Lot arachide & Lot colza & Lot hareng & Lot hareng + maïs \\
\hline Nombre de rats & 5 & 5 & 4 & 5 \\
\hline MS ingérée $\ldots$. & $83,6 \pm 3,4$ & $85,8 \pm 3,9$ & $95,2 \pm 1,9 \quad$ (l) & $95,8 \pm 4,1$ \\
\hline MS excrétée .... & $4,00 \pm 0,14$ & $4,52 \pm 0,23$ & $5,00 \pm 0,33(\mathbf{1})$ & $4,58 \pm 0,26$ \\
\hline CUD de la MS .... & $95,2 \pm 0,2$ & $94,7 \pm 0,3$ & $94,8 \pm 0,3$ & $95,2 \pm 0,2$ \\
\hline LT ingérés $\ldots \ldots \ldots$ & $12,5 \pm 0,5$ & $12,9 \pm 0,5$ & $14,0 \pm 0,2$ & $14,4 \pm 0,6$ \\
\hline LT excrétés ...... & $1,26 \pm 0,13$ & $2,08 \pm 0,09\left({ }^{2}\right)$ & $2,12 \pm 0,14\left({ }^{2}\right)$ & $1,68 \pm 0,09\left({ }^{1}\right) *$ \\
\hline CUD des LT.. & $89,9 \pm 1,1$ & $83,7 \pm 1,1 \quad\left({ }^{2}\right)$ & $84,9 \pm 0,7$ & $88,3 \pm 0,6^{* *}$ \\
\hline $\mathrm{AG}$ ingérés ... & $11,7 \pm 0,5$ & $12,0 \pm 0,6$ & $13,1 \pm 0,2$ & $13,3 \pm 0,6$ \\
\hline AG excrétés .... & $0,92 \pm 0,13$ & $1,76 \pm 0,15\left({ }^{2}\right)$ & $1,85 \pm 0,11\left(^{2}\right)$ & $1,44 \pm 0,07\left(^{1}\right)^{*}$ \\
\hline CUD des $A G \ldots \ldots$ & $92,0 \pm 1,2$ & $85,2 \pm 1,5 \quad\left({ }^{2}\right)$ & $\left.85,9 \pm 0,7 \quad{ }^{2}\right)$ & $89,2 \pm 0,5^{* *}$ \\
\hline
\end{tabular}

(1) Différence significative avec le lot arachide au seuil de $\mathrm{P}=0,05$.

(2) Différence significative avec le lot arachide au seuil de $\mathrm{P}=0,01$.

* : Différence significative avec le lot hareng au seuil de $\mathrm{P}=0,05$.

** : Différence significative avec le lot hareng au seuil de $\mathrm{P}=0,01$.

L'analyse des acides gras fécaux permet de calculer le CUD apparent des principaux d'entre eux, pour chacun des 4 régimes (tabl, 2). Il est ainsi confirmé que, chez les rats ingérant de l'huile de colza, les acides gras fécaux sont principalement constitués d'acide érucique (8I p. Ioo) dont le CUD assez bas (75 p. Ioo) rend compte de la médiocre utilisation digestive de cette huile. Le cas de l'huile de hareng est différent. Les acides gras à longue chaîne $\mathrm{C}_{20}:_{1}$ et $\mathrm{C}_{22}:{ }_{1}$ contribuent, par leurs CUD relativement bas ( $8 \mathrm{I}$ et $76 \mathrm{p}$. Ioo respectivement), à abaisser l'utilisation digestive de

(1) CUD apparent $=-\frac{\text { lipides (ou ac. gras) ingérés }- \text { lipides (ou ac. gras) excrétés }}{\text { lipides (ou ac. gras) ingérés }} \times \frac{\text { go. }}{\text { gra }}$ 


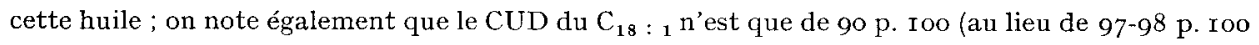
pour le $\mathrm{C}_{18}: 1$ des huiles d'arachide et de colza) ; les acides gras trans ont un CUD de 87 p. Ioo, voisin de celui de l'ensemble des acides gras de l'huile. Mais on remarque surtout que les compositions en acides gras des lipides fécaux des lots hareng et hareng + maïs sont très semblables, et que, par conséquent, l'addition d'huile de maïs à l'huile de hareng améliore le CUD de tous les acides gras de l'huile, y compris celui des acides gras à longue chaîne $\mathrm{C}_{20}:_{1}$ et $\mathrm{C}_{22}:{ }_{1}$. Ceux-ci ne constituent donc pas, dans le cas présent, le seul facteur limitant l'utilisation digestive de l'huile de hareng.

La composition des huiles de poissons partiellement hydrogénées étant très variable, notamment du point de vue des teneurs en $\mathrm{C}_{18}: 2$ (selon le degré d'hydrogénation), en $\mathrm{C}_{20}:{ }_{1}$ et en $\mathrm{C}_{22}: \mathbf{1}$ (qui peuvent atteindre, ensemble, plus de 50 p. roo des acides gras de l'huile), les résultats rapportés dans la présente note ne peuvent être généralisés sans réserves.

Reçu pour publication en janvier 1975.

\section{REMERCIEMENTS}

Ces recherches ont été poursuivies avec l'aide financière de 1'I. N. S. E. R. M. (action thématique programmée $n^{\circ} 6$ ). Les huiles nous ont été fournies par les Sociétés Lesieur-Cotelle et Astra-Calvé, et par l'Institut des Corps gras.

\section{SUMMARY}

\section{DIGESTIBILITY OF PARTIALLY HYDROGENATED HERRING OIL IN THE RAT}

Apparent digestibility of partially hydrogenated herring oil (containing 13 p. Ioo of $\mathrm{C}_{22}:{ }_{1}$ ) is lower than peanut oil. It is improved by adding corn oil.

\section{RÉFÉRENCES BIBLIOGRAPHIQUES}

A. O. C. S. Tentative Method Cd I4-6I, I973. Isolated Trans Isomers, Infrared Spectrophotometric Method. Rocquelin G., Leclerc J., ig69. L'huile de colza riche en acide érucique et l'huile de colza sans acide érucique. II. Utilisation digestive comparée chez le Rat. Ann. Biol. anim. Bioch. Biophys,, 9, 4I3-426.

Savary P., Constantin J., ig66. Sur la résorption intestinale des chaines éruciques et leur incorporation dans les chylomicrons lymphatiques du Rat. Biochem. Biophys. Acta, 125, II8-I 28. 